\title{
ASYMMETRIC AND SYMMETRIC GRAPHS
}

\author{
by E. M. WRIGHT $\dagger$
}

(Received 2 December, 1972)

An $(n, q)$ graph consists of $n$ nodes and $q$ edges, i.e. $q$ distinct unordered pairs of different nodes, so that there are no loops or multiple edges. We write $T$ for the number of unlabelled $(n, q)$ graphs and $F$ for the number of labelled $(n, q)$ graphs. We say that a labelled graph is symmetric if there is a nonidentical permutation of its nodes which leaves the graph unaltered. We write $r$ for the order of the automorphism group of the graph, i.e. the group of all those permutations of the nodes which leave the graph unaltered; we say that the graph is of symmetry order $r$. A graph which is not symmetric is called asymmetric and, for such a graph, obviously $r=1$. We say that an unlabelled graph is symmetric or asymmetric according as the graph obtained by labelling its nodes is symmetric or asymmetric.

We write $N=n(n-1) / 2$ and $B(h, k)=h ! /\{k !(h-k) !\}$. Then $0 \leqq q \leqq N$ and $F=B(N, q)$. If an $(n, q)$ graph is symmetric of order $r$, then so is its complement, i.e. the $(n, N-q)$ graph which has just those edges that the original graph lacks. Hence we can take $0 \leqq q \leqq(N / 2)$ without loss of generality. We write $\mu=(2 q-n \log n) / n$. We write $C$ for a positive number, not always the same at each occurrence, independent of $n$ and $q$. The notations $O()$ and $o($ ) refer to the passage of $n$ to infinity and each constant implied is a $C$. If we say that " almost all" graphs of a particular class have property $P$, we mean that the ratio of the number of those which lack the property to the number of those which have the property tends to 0 as $n \rightarrow \infty$. All our statements carry the implied condition that $n>C$.

Erdös and Renyi [1] considered labelled asymmetric graphs and, amongst other results, showed that almost all labelled graphs on $n$ nodes are asymmetric. They announced the further result that, if $\mu \rightarrow \infty$, then almost all labelled $(n, q)$ graphs are asymmetric.

We write $T(r)$ for the number of unlabelled $(n, q)$ graphs of symmetry order $r$ and $F(r)$ for the corresponding number of labelled graphs. Clearly

$$
n ! T(r)=r F(r) \text {. }
$$

We write $T^{(a)}$ (resp. $T^{(s)}$ ) for the number of unlabelled asymmetric (resp. symmetric) $(n, q)$ graphs and $F^{(a)}$ (resp. $F^{(s)}$ ) for the numbers of labelled asymmetric (resp. symmetric) $(n, q)$ graphs, so that

$$
F^{(a)}=F(1), \quad F^{(s)}=\sum_{r=2}^{n !} F(r), \quad T^{(a)}=T(1), \quad T^{(s)}=\sum_{r=2}^{n !} T(r) .
$$

Let $F_{\pi}$ be the number of labelled $(n, q)$ graphs which are invariant under the permutation $\pi$ of the $n$ labelled nodes. The identity permutation is $I$, so that $F_{I}=F$. By the Polya-Burnside Counting Theorem [4], we have

$$
n ! T=\sum_{n} F_{\pi}=F+S \quad\left(S=\sum_{\pi \neq I} F_{\pi}\right),
$$

$\dagger$ The research reported herein was sponsored in part by the United States Government. 
where the first sum is taken over all the $n$ ! possible permutations $\pi$ of the $n$ labelled nodes. We have

and so, by (1),

$$
F=\sum_{r=1}^{n !} F(r), \quad T=\sum_{r=1}^{n !} T(r)
$$

$$
S=\sum_{r=2}^{n !}(r-1) F(r)=n ! \sum_{r=2}^{n !}(r-1) T(r) / r .
$$

We require two lemmas.

LEMMA 1. If $\mu \rightarrow \infty$, then $S=o(F)$.

LEMMA 2. If $\mu \leqq 0$, then $F=o(S)$.

I proved Lemma 1 in [6] (in fact, I showed that $S=o(F)$ if and only if $\mu \rightarrow \infty$ ). I prove Lemma 2 later in the present paper.

THEOREM 1. If $\mu \rightarrow \infty$, then almost all labelled $(n, q)$ graphs are asymmetric; i.e.,

$$
F^{(s)}=o\left(F^{(a)}\right) \text {. }
$$

This is the theorem announced by Erdös and Renyi [1]. It follows at once from Lemma 1, since we have

$$
\begin{aligned}
F^{(s)} & =\sum_{r=2}^{n l} F(r) \leqq \sum_{r=2}^{n l}(r-1) F(r) \\
& =S=o(F)=o\left(F^{(a)}+F^{(s)}\right) .
\end{aligned}
$$

THEOREM 2. If $\mu \rightarrow \infty$, then almost all unlabelled $(n, q)$ graphs are asymmetric; i.e.,

We have

$$
T^{(s)}=o\left(T^{(a)}\right)
$$

$$
\begin{aligned}
n ! T^{(s)} & =n ! \sum_{r=2}^{n !} T(r) \leqq 2(n !) \sum_{r=2}^{n !}(r-1) T(r) / r \\
& =2 S=o(F)=n ! o(T)
\end{aligned}
$$

and so (4). Since

$$
F^{(a)} / F^{(s)} \geqq 2 T^{(a)} / T^{(s)}
$$

by (1), Theorem 2 implies Theorem 1 .

THEOREM 3. If $\mu \leqq 0$, then almost all unlabelled $(n, q)$ graphs are symmetric; i.e.,

We have

$$
T^{(a)}=o\left(T^{(s)}\right) \text {. }
$$

by Lemma 2 and

$$
n ! T^{(a)}=n ! T(1)=F(1) \leqq F=o(S)
$$

by (2). The theorem follows.

$$
S \leqq n ! \sum_{r=2}^{n} T(r)=n ! T^{(s)}
$$


THEOREM 4. If $\mu \leqq 0$, then, for any fixed $R$, almost all unlabelled $(n, q)$ graphs are of symmetry order greater than $R$.

For, by Lemma 2,

$$
\begin{aligned}
n ! \sum_{r=1}^{R} T(r) & \leqq R \sum_{r=1}^{R} n ! T(r) / r=R \sum_{r=1}^{R} F(r) \\
& \leqq R F=o(S)=o(n ! T) .
\end{aligned}
$$

THEOREM 5. If $\mu \rightarrow-\infty$ as $n \rightarrow \infty$, then almost all labelled $(n, q)$ graphs are symmetric; i.e., $F^{(a)}=o\left(F^{(s)}\right)$.

I conjecture that the conditions in Theorems 3 and 5 are necessary as well as sufficient but I am unable to prove this. What I can prove however is the following theorem, which shows that the conditions of Theorems 1 and 2 are necessary as well as sufficient.

THEOREM 6. If $\mu$ is bounded above as $n \rightarrow \infty$, then $F^{(s)} \neq o\left(F^{(a)}\right)$ and $T^{(s)} \neq o\left(T^{(a)}\right)$.

Before proving Theorem 5 it is convenient to prove Lemma 2, since a subsidiary lemma is needed to prove both.

Proof of Lemma 2. Lemma 2 can be deduced from an asymptotic approximation to $T$ which I announced in [7] and indeed the result can be seen to be true under the slightly wider condition that $\overline{\lim } \mu \leqq 0$. Hence Theorem 3 is true under this wider condition. But the calculations leading to this approximation are very much more elaborate than the proof of Lemma 2 which I give here.

Let $p$ be the number of nodes unchanged by the permutation $\pi$. Then an $(n, q)$ graph composed of any $(p, q)$ graph on these $p$ nodes and the other $n-p$ isolated nodes is invariant under $\pi$. Hence

$$
F_{n} \geqq F(p, q)=B(P, q),
$$

where $P=p(p-1) / 2$. The $p$ unchanged nodes may be chosen in $B(n, p)$ ways and, when these are chosen, there are $H_{1}(n-p)$ ways of permuting the remaining $n-p$ nodes, where $H_{1}(n)$ is Euler's rencontre number, i.e. the number of ways of permuting $n$ different objects so that none remains unmoved. Hence there are just

$$
B(n, p) H_{1}(n-p)
$$

different $\pi$ which leave just $p$ nodes unchanged.

We have then

$$
S \geqq \sum_{p=0}^{n-2} H_{1}(n-p) B(n, p) B(P, q) .
$$

It was proved by Euler $[3,5]$ that

$$
H_{1}(n)=(n-1)\left\{H_{1}(n-1)+H_{1}(n-2)\right\}
$$

and, from this, we can prove by induction on $n$ that

$$
H_{1}(n) \geqq C(n !) \quad(n \geqq 2) .
$$


Hence, if we write $t=n-p$ and

we have

$$
\Omega_{\imath}=B(P, q) / p !
$$

$$
S / F>C \sum_{t=2}^{n} \Omega_{t} / \Omega_{0}
$$

We write $j=\left[n^{1 / 2} / \log n\right]$. A little calculation suffices to deduce the following lemma from Stirling's Theorem and the Second Mean Value Theorem.

Lemma 3. If $\mu<C$ and $0 \leqq t \leqq j$, then

$$
\Omega_{t} \sim \Omega_{0} e^{-\mu t}
$$

as $n \rightarrow \infty$.

If $\mu \leqq 0$, we deduce that

$$
S / F \geqq C \sum_{i=2}^{j} e^{-\mu t} \rightarrow \infty
$$

as $n \rightarrow \infty$. This is Lemma 2 .

Proof of Theorem 5. We write $L=L(n, q)$ for the number of labelled $(n, q)$ graphs which contain at least 2 isolated nodes and so are necessarily symmetric. Again $f=f(n, q)$ is the number of connected labelled $(n, q)$ graphs. Clearly

Next

$$
F^{(s)} \geqq L(n, q) \text {. }
$$

$$
L(n, q) \geqq \sum_{t=2}^{n} B(n, t) f(n-t, q),
$$

since the typical term on the right enumerates the number of labelled $(n, q)$ graphs that consist of $t$ isolated nodes and a connected $(n-t, q)$ graph.

Erdős and Renyi [2] showed that, for bounded $\mu$, we have

$$
f(n, q) / F(n, q) \sim \exp \left(-e^{-\mu}\right) .
$$

Hence, if $2 \leqq t \leqq j$, we have

where

$$
\begin{aligned}
f(n-t, q) & \geqq F(n-t, q)\left\{\exp \left(-e^{-\mu^{\prime}}\right)+o(1)\right\}, \\
& \geqq F(n-t, q)\left\{\exp \left(-e^{-\mu}\right)+o(1)\right\},
\end{aligned}
$$

if $t>0$. Hence

where

$$
\mu^{\prime}=\{2 q /(n-t)\}-\log (n-t)>\mu
$$

by Lemma 3. Hence

$$
L \geqq F E\left\{\exp \left(-e^{-\mu}\right)+o(1)\right\}
$$

$$
E=\sum_{t=2}^{j} B(n, t) B(P, q) / B(N, q) \sim \sum_{t=2}^{j} e^{-\mu t} / t !
$$

$$
E \sim \sum_{t=2}^{\infty} e^{-\mu t} / t !=\exp \left(e^{-\mu}\right)-1-e^{-\mu}
$$


and so

$$
L / F \geqq\left\{1-\left(1+e^{-\mu}\right) \exp \left(-e^{-\mu}\right)\right\}\{1+o(1)\} .
$$

This is true for bounded $\mu$. But it is easy to show that $L / F$, the proportion of labelled $(n, q)$ graphs which contain at least two isolated nodes, decreases (at least non-strictly) as $q$ increases for fixed $n$. Hence, if $\mu \rightarrow-\infty$ as $n \rightarrow \infty$, we have $L / F \rightarrow 1$. Hence, by (6), $F^{(s)} / F \rightarrow 1$ and this is Theorem 5 .

Again, if $\mu \rightarrow c$, a fixed finite number, as $n \rightarrow \infty$, we see that

$$
1-\left(1+e^{-\mu}\right) \exp \left(-e^{-\mu}\right) \rightarrow 1-\left(1+e^{-c}\right) / \exp \left(e^{-c}\right)>0 \text {. }
$$

Hence $F^{(s)} / F$ does not tend to zero, nor, by (5) does $T^{(s)} / T^{(a)}$. Hence Theorem 6 .

\section{REFERENCES}

1. P. Erdös and A. Renyi, Asymmetric graphs, Acta Math. Acad. Sci. Hungar. 14 (1963), 295-315, especially 298.

2. P. Erdös and A. Renyi, On random graphs I, Publ. Math. Debrecen 6 (1959), 290-297.

3. L. Euler, Solutio questionis curiosae ex doctrina combinationum, Mem. Acad. Sci. St. Petersbourg 3 (1811), 57-64; Opera omnia (1) 7 (1923), 435-448.

4. G. Polya, Kombinatorische Anzahlbestimmungen für Gruppen, Graphen und Chemische Verbindungen, Acta Math. 68 (1937), 145-254.

5. J. Riordan, An introduction to combinatorial analysis (New York, 1958), 57-62.

6. E. M. Wright, Graphs on unlabelled nodes with a given number of edges, Acta Math. 126 (1971), 1-9.

7. E. M. Wright, The number of unlabelled graphs with many nodes and edges, Bull. Amer. Math. Soc. 78 (1972), 1032-1034.

\section{UNIVERSITY OF ABERDEEN}

